\title{
CDK1 plays an important role in the maintenance of pluripotency and genomic stability in human pluripotent stem cells
}

\author{
I Neganova ${ }^{1}$, K Tilgner $^{1}$, A Buskin ${ }^{1}$, I Paraskevopoulou ${ }^{1}$, SP Atkinson ${ }^{1}$, D Peberdy ${ }^{1}$, JF Passos ${ }^{2}$ and M Lako ${ }^{\star, 1}$
}

Human embryonic stem cells (hESC) and induced pluripotent stem cells (hiPSC) are characterised by an unusual and tightly regulated cell cycle that has been shown to be important for the maintenance of a pluripotent phenotype. Cyclin-dependant kinase 1 (CDK1) is a key player in cell cycle regulation and particularly mitosis; however, its role has not been studied previously in hESC and hiPSC. To investigate the impacts of CDK1 downregulation, we performed RNA interference studies which in addition to expected mitotic deficiencies revealed a large range of additional phenotypes related to maintenance of pluripotency, ability to repair double strand breaks (DSBs) and commitment to apoptosis. Downregulation of CDK1 led to the loss of typical pluripotent stem cell morphology, downregulation of pluripotency markers and upregulation of a large number of differentiation markers. In addition, human pluripotent stem cells with reduced CDK1 expression accumulated a higher number of DSBs were unable to activate CHK2 expression and could not maintain G2/M arrest upon exposure to ionising radiation. CDK1 downregulation led to the accumulation of cells with abnormal numbers of mitotic organelles, multiple chromosomal abnormalities and polyploidy. Furthermore, such cells demonstrated an inability to execute apoptosis under normal culture conditions, despite a significant increase in the expression of active PARP1, resulting in tolerance and very likely further propagation of genomic instabilities and ensuing of differentiation process. On the contrary, apoptosis but not differentiation, was the preferred route for such cells when they were subjected to ionising radiation. Together these data suggest that CDK1 regulates multiple events in human pluripotent stem cells ranging from regulation of mitosis, G2/M checkpoint maintenance, execution of apoptosis, maintenance of pluripotency and genomic stability.

Cell Death and Disease (2014) 5, e1508; doi:10.1038/cddis.2014.464; published online 6 November 2014

The high proliferation ability and maintenance of pluripotency in human pluripotent stem cells is directly linked to the regulation of core cell cycle factors. ${ }^{1-7}$ Human embryonic stem cell (hESCs) are derived from the inner cell mass of preimplantation blastocysts, whereas induced pluripotent stem cells (hiPSCs) are generated from the reprogramming of somatic cells back to pluripotency. Both cell types have the potential to generate almost any cell type of the human adult organism and for this reason, it is assumed that the requirement for genomic stability is critical; however, chromosomal instabilities are often observed in hESCs and hiPSCs. $^{8-10}$

With the aim to better understand mitotic progression and its regulation in human pluripotent stem cells, we focused on a key cell cycle regulator, cyclindependent kinase 1 (Cdk1). ${ }^{11}$ Although the majority of Cdks and cyclins have been shown to be largely dispensable, Cdk1 has emerged as the master regulator of mammalian cell cycle, whose role in vivo cannot be compensated by other closely related Cdks, including Cdk2. ${ }^{12-14}$ Studies performed in primary cultures and established cell lines do not always match up to the wide range of Cdk and Cyclin compensatory mechanisms observed in vivo and this has led to the idea that certain cell types (especially cells emerging during embryonic development) may have not developed the full spectrum of compensatory mechanisms. ${ }^{15,16}$

Cdk1 is highly expressed in murine ESCs and interacts directly with Oct4, enhancing its binding to the trophoectoderm marker $C d x 2$ and promoting its repression. ${ }^{17}$ Knockdown of Cdk1 relieves this repression, resulting in the activation of $C d x 2$ and differentiation of mouse ESCs into trophoectodermal lineages. ${ }^{17,18}$ Conditional knock-out of Cdk1 in mouse results in the arrest of embryonic development around the blastocyst stage and DNA re-replication, because of an increase in Cdk2/Cyclin A activity. ${ }^{14}$ Similarly, the inhibition of Cdk1 via a chemical inhibitor (R03306) leads to abortive endoreduplication and apoptosis in murine ESCs, ${ }^{19}$ suggesting an important role for this kinase in mitotic progression in ESCs. Till now, insights on CDK1 function in hESC and hiPSC are missing, despite $\mathrm{CDK} 1 / 2$ emerging as the central kinome

${ }^{1}$ Institute of Genetic Medicine, Newcastle University, International Centre for Life, Newcastle upon Tyne NE1 3BZ, UK and ${ }^{2}$ Centre for Integrated Systems Biology of Ageing and Nutrition, Institute for Ageing and Health, Newcastle University, Newcastle upon Tyne NE4 5PL, UK

*Corresponding author: M Lako, Institute of Human Genetics/Newcastle University, Newcastle upon Tyne, UK. Tel: +44191 2418688; Fax: +44191 2418666; E-mail: Majlinda.Lako@ncl.ac.uk

Abbreviations: hESC, Human embryonic stem cells; hiPSC, induced pluripotent stem cells; DSBs, double strand breaks; siRNAs, small interfering RNAs; AP, alkaline phosphatase; IR, ionising irradiation; HR, homologous recombination; TUNEL, Terminal deoxynucleotidyl transferase dUTP nick end labeling; BrdU, 5-bromo-2deoxyuridine; PE, Phycoerythrin; DAPI, 4',6-diamidino-2-phenylindole

Received 03.6.14; revised 16.9.14; accepted 24.9.14; Edited by D Aberdam 
controlling self-renewal and differentiation of these cells. ${ }^{20}$ The high proliferative nature of hESC and hiPSC would suggest a high dependence of these cells on CDK1 for the proper regulation of mitosis and successful completion of cell cycle. However, how CDK1 regulates and/or impacts other processes, for example, apoptosis, maintenance of pluripotency and genomic stability is unknown and forms the main focus of this manuscript.

\section{Results}

Downregulation of CDK1 causes pluripotent stem cell accumulation in G2 phase, loss of pluripotency and induction of differentiation. Our western blot analysis of hESC synchronised at different stages of the cell cycle shows that although total CDK1 expression does not vary through the cell cycle, the expression of the phosphorylated form (Thr161), which is required for the activation of the CDK1CYCLIN B1 complex, is highest in S and G2 phases of the cell cycle (Figure 1a). In contrast, the expression of the Tyr15/ Thr14 phosphorylated form, which results in CDK1 inactivation, is lower in S and G2 (Figure 1a). Together, these data suggest highest expression of active CDK1 in S and G2 phases of hESC cell cycle. Immunoprecipitation analysis indicates that CDK1 forms complexes with CYCLIN B1 and CYCLIN A which are key players in the regulation of mitosis (Figure 1b). Immunofluorescence analysis (with Ki67 and CDK1 antibodies) to distinguish the specific stages of the cell cycle (performed as in Becker et al. ${ }^{21}$ ) indicated a high nuclear CDK1 accumulation and a low cytoplasmic expression pattern (Figure 1c) during late G1/S and S-phase/G2 transition in hESC.

To further investigate the role of CDK1 in hESC and hiPSC, we performed RNA interference studies using small interfering RNAs (siRNAs). Combined quantitative reverse transcriptionpolymerase chain reaction (qRT-PCR) analysis (Figure 2a) and western blotting (Figure $2 \mathrm{~b}$, Supplementary Figure $1 \mathrm{~A}$ ) indicated effective CDK1 downregulation as early as day 1 post transfection of CDK1-siRNAs. Immunocytochemical analysis revealed the complete loss of cytoplasmic CDK1 expression and a very significant reduction of nuclear CDK1 expression in CDK1 siRNA-transfected cells (Supplementary Figure 2), when compared with control cells. Furthermore, we observed changes in the location of CYCLIN B1 expression (Supplementary Figure 3). We noticed that CYCLIN B1 in hESC is localized at the nuclei of control cells (Supplementary Figure 3); however, in the CDK1 siRNA group, CYCLIN B1 was observed both at cytoplasm and the nuclei, suggesting a requirement for CDK1 activity in nuclear translocation of CYCLIN B1 in pluripotent stem cells similarly to observations made in human somatic cells. ${ }^{22,23}$

$C D K 1$ downregulation resulted also in cell cycle changes with hESC accumulation in G2 phase at the expense of G1 and $S$ phases (Figures $2 \mathrm{c}$ and $\mathrm{d}$ ). Similar experiments performed in hiPSC indicated the accumulation of cells in G2 phase at the expense of S phase; however, no changes in G1 phase were observed highlighting some differences between hESC and hiPSC (Supplementary Figure 1B,C). Despite this, we observed the loss of typical pluripotent stem cell morphology upon $C D K 1$ downregulation in both cell types (Figure 2e, Supplementary Figure 1D). Expression analysis indicated a reduction in expression of three pluripotency a

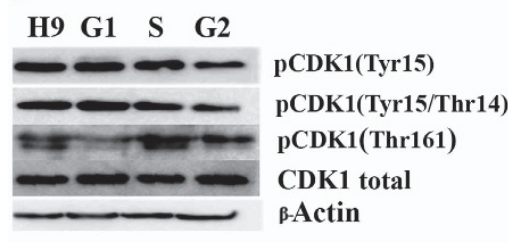

b

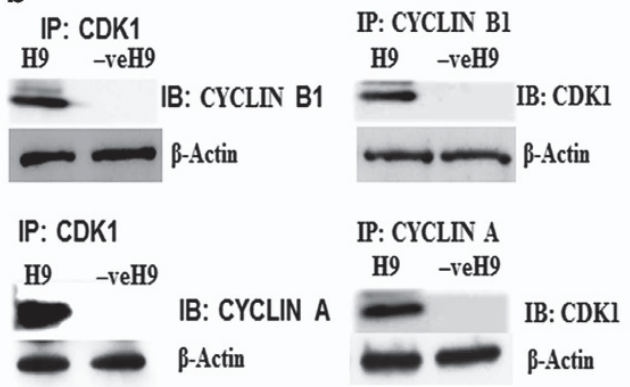

c

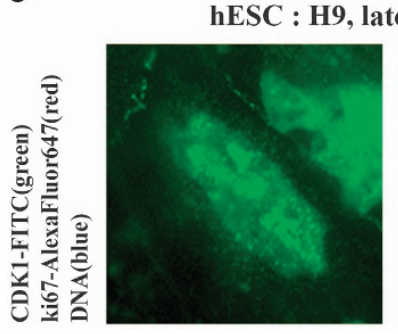

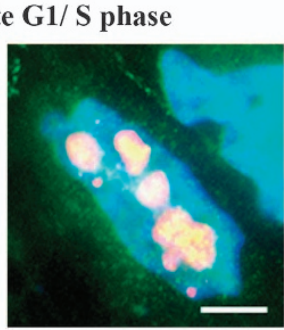

late $\mathrm{S}$ phase /G2

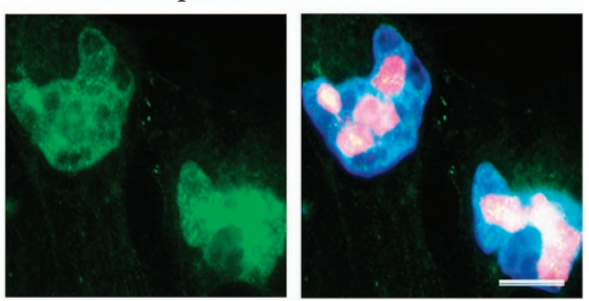

Figure 1 Expression and cellular localization of CDK1 in hESC. (a) Western blot of a total hESC lysates probed with antibodies against total CDK1 and specific phosphoresidues important for cell cycle progression. Cells were synchronized at G1 (70\%), S (75\%) and G2 (68\%) phases of the cell cycle as described before at Neganova et al.: ${ }^{2}$ H9-unsynchronized population. (b) Analysis of specific complex formation between CDK1/Cyclin B1 and CDK1/Cyclin A via immunoprecipitation in unsynchronized hESC. (c) CDK1 cellular localisation in H9 hESC during transition from late G1 to S phase and late S/G2 phase transition revealed by indirect immunofluorescence with anti-CDK1 antibody (green) and anti-Ki67 (red). Scale bar $=10 \mu \mathrm{m}$. Presented images are representative of at least three independent experiments 

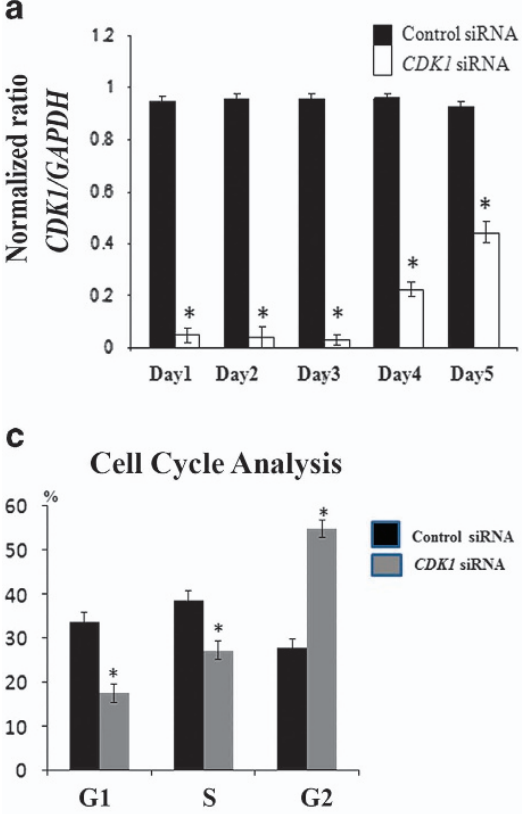

b

Day1 Day2 Day3 Day4 ControlCDK1ControlCDK1Control CDK1Control CDK1

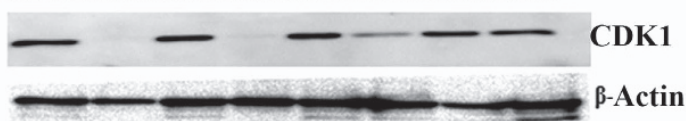

d

Control siRNA
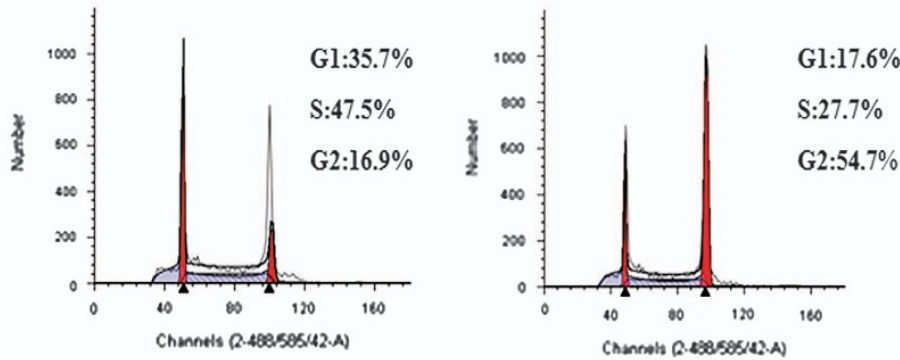

e

Day2
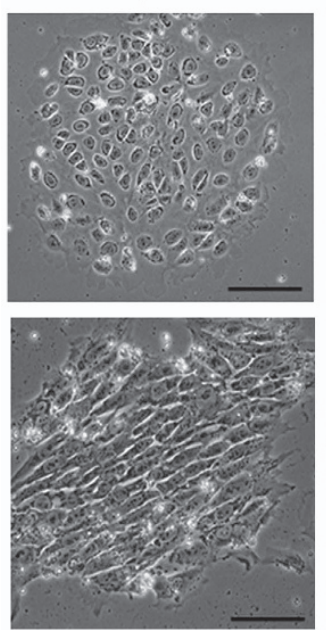

Day3
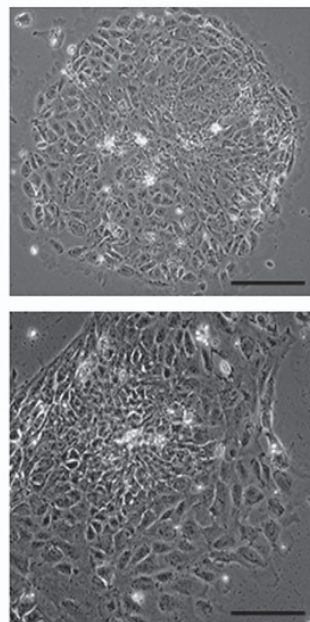

Day4

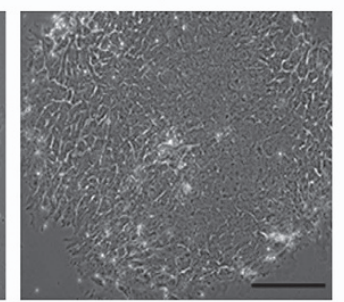

Control siRNA

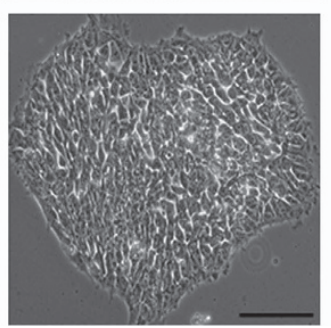

CDK1 SIRNA

Figure 2 Downregulation of CDK1 by RNAi effectively abrogates CDK1 expression and induces the accumulation of $h E S C$ at the G2 phase of the cell cycle. (a) Quantitative RT-PCR analysis for expression of CDK1 from 24 to $120 \mathrm{~h}$ post transfection. Results are presented as average + S.E.M. $(n=3)$. T-test analysis was carried out to assess differences in gene expression between the control and CDK1 siRNA group, ${ }^{*} P<0.05$. (b) Western blot analysis of CDK1 expression. $\beta$-Actin was used as the loading control. The data shown are representative of six independent western blots carried out in $\mathrm{H} 9 \mathrm{hESC}$ line. (c) Graphical representation of the ModFit analysis of cell cycle distribution in the control and CDK1 siRNA group, $48 \mathrm{~h}$ post transfection with siRNAs. Results are presented as average \pm S.E.M. $(n=6)$. T-test analysis was carried out to assess the differences in gene expression between the control and CDK1 siRNA group, ${ }^{*} P<0.05$. (d) Flow cytometric histograms showing cell cycle distribution in control and $C D K 1$ siRNA groups. The percentage of cells in each stage of the cell cycle is indicated in the top right corner of flow histogram. Data are representative of at least six independent experiments. (e) Phasecontrast images of hESC transfected with control and CDK1 siRNAs (upper and lower panel respectively) at 2, 3 and 4 days post transfection. Scale bar $=25 \mu \mathrm{m}$

markers, namely OCT4, KLF4 and LIN28 (Figures 3a and c), and upregulation of $C D X 2$ (trophoectodermal marker), PAX6 and NESTIN (ectodermal markers), FGF5 (primitive ectoderm marker), BRACHYURY (mesodermal marker) and AFP (endodermal marker), suggesting the loss of pluripotency upon CDK1 knockdown (Figure 3b). This was also confirmed by alkaline phosphatase (AP) staining for almost complete loss of $\mathrm{AP}+$ colonies in the CDK1 siRNA group that was observed when compared with control siRNA-transfected group which contained on average about $80 \% \mathrm{AP}+$ colonies
(Figure 3d, Supplementary Figure 1D). Counting of cell nuclei also showed that a considerable proportion of cells $(\sim 14 \%)$ in the CDK1-siRNA transfected group became polyploid (Figures $3 e$ and f; Supplementary Figure 1D). Cytogenetic analysis revealed the presence of abnormal and multicentric chromosomes in addition to loss and gain of whole chromosomes in $100 \%$ of metaphases obtained from the CDK1 siRNA group, whereas control cells showed normal karyotype (Supplementary Figure 4). Together, these data indicate an important role for CDK1 in cell cycle regulation, maintenance 
a
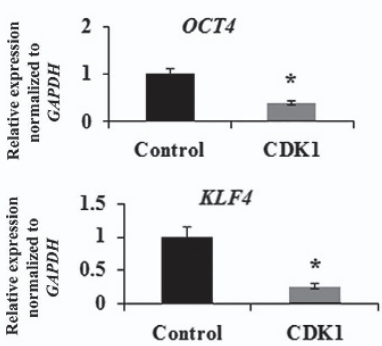

LIN28

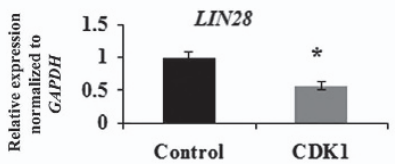

c

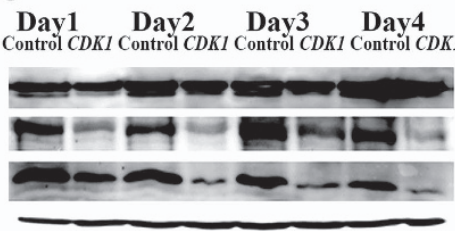

OCT4

KLF4

LIN28

GAPDH

e

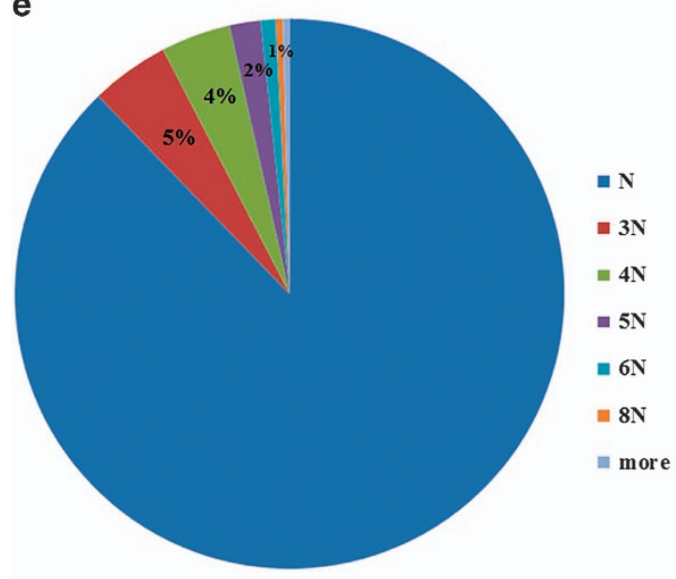

$C D X 2$
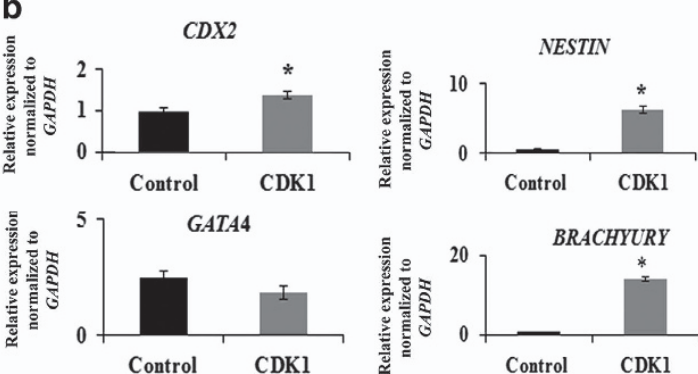

PAX6
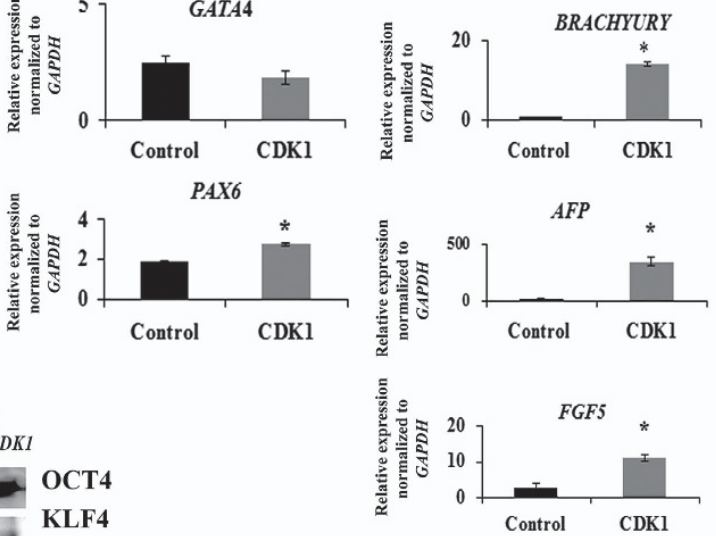

d

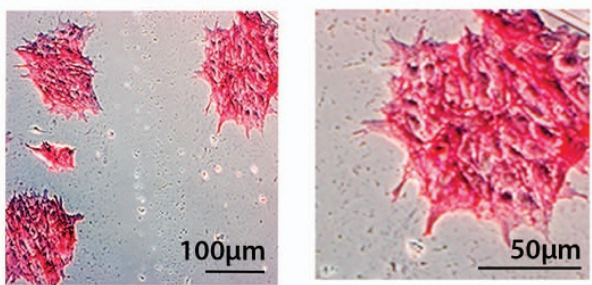

CDK1 siRNA
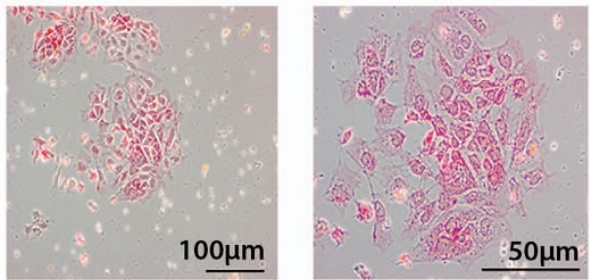

f

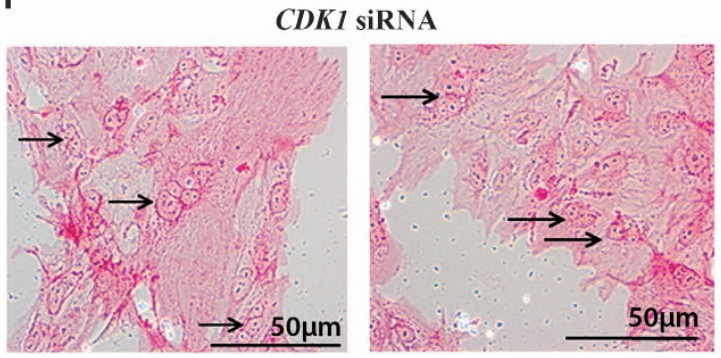

Figure 3 Downregulation of CDK1 leads to the loss of pluripotency, differentiation and polyploidy. (a) Expression analysis of main pluripotency genes by quantitative RT-PCR. Results are presented as average \pm S.E.M. $(n=3)$. The value for the $h E S C$ transfected with control siRNA was set to 1 and all other values were calculated with respect to that. $T$-test analysis was carried out to assess the differences in gene expression between the control and CDK1 siRNA group, ${ }^{*} P<0.05$. (b) Upregulation of differentiation markers analysed by quantitative RT-PCR. Results are presented as mean \pm S.E.M. $(n=3)$. The value for the $h E S C$ transfected with control siRNA was set to 1 and all other values were calculated with respect to that. $T$-test analysis was carried out to assess the differences in gene expression between the control and $C D K 1$ siRNA group, ${ }^{*} P<0.05$. (c) Western blot analysis for the expression of key pluripotency markers. Note that the protein level of KLF4 and LIN28 was not restored by day 4 post transfection. GAPDH was used as a loading control. Data are representative of at least three independent experiments. (d) Alkaline-phosphatase-positive staining was observed in hESC transfected with control siRNA (upper row) but differentiated morphology and lack of typical staining was observed in cells transfected with CDK1 siRNAs (lower panel), $48 \mathrm{~h}$ post transfection. Images are representative of at least three independent experiments. (e) Representative histogram showing the percentage of polyploid cells in the CDK1 siRNA group at $48 \mathrm{~h}$ post transfection. At least 300 cells were analysed in each experiment. (f) Examples of appearance of multinucleated cells (black arrows) on a second day after CDK1siRNA treatment. Scale bar $=50 \mu \mathrm{m}$ 
of pluripotency and genomic stability in human pluripotent stem cells.

Downregulation of CDK1 results in accumulation of double strand breaks (DSBs) and impairment of CHK2 activation. Under normoxic culture conditions, hESC accumulate a small number of DSBs (Figures $4 a$ and $b^{3}{ }^{3}$ ). However, upon $C D K 1$ knockdown, we observed a significant increase both in the percentage of hESC with DSBS (Figure $4 \mathrm{a}$ ) and the number of DSB foci per cell (Figures $4 \mathrm{a}$ and b), corroborating data previously published in mammalian somatic cells. ${ }^{24,25}$ To further confirm this, we carried out $\gamma$-H2A.X detection by flow cytometric analysis (Figure 4c). It is evident that upon $C D K 1$ downregulation, there is a significant increase in the number of accumulated DSBs (Figures $4 \mathrm{a}$ and $\mathrm{c}$ ); however, this is not accompanied by increased apoptosis in the CDK1 siRNA group when compared with the control (Figure 7d). When analysed under the context of cell cycle regulation, $59 \%$ of S-phase cells were positive for $\gamma$-H2A.X, in contrast to control cells which showed only $6.7 \%$ of S-phase cells with $\gamma$-H2A.X foci (Figure 4c). We repeated the same analysis at $16 \mathrm{~h}$ after administrating ionising radiation (IR) (2Gy) to hESC (Figure 4c). Although there is a slightly higher DSBs accumulation in hESC with reduced CDK1 expression under IR when compared with the same group under non-IR conditions (Figure 4c), the pattern is similar with the majority of cells with $\gamma$-H2A.X in the $S$ phase of the cell cycle.

Next, we investigated whether downregulation of CDK1 affects DNA damage response signalling. We observed a slight upregulation of CHK1 (day 1-3 post transfection, Figure 4d); however, there was a very significant downregulation of $\mathrm{CHK} 2$ up to $48 \mathrm{~h}$ post transfection of $C D K 1$, suggesting an important link between CDK1 function and its ability to maintain intact CHK2 expression. This effect is also observed upon the administration of IR) which leads to a significant increase in the expression of the phosphorylated form of CHK1 (Ser 345), but not the phosphorylated form of CHK2 (Thr368, Figure 4e) following the downregulation of CDK1. p53 has been shown to be activated by both $\mathrm{CHK} 2$ and $\mathrm{CHK} 1$ in response to DNA damage; however, the specificity of $\mathrm{CHK} 1$ versus $\mathrm{CHK} 2$ activation can be distinguished because $\mathrm{CHK} 2$-dependent activation of $\mathrm{p} 53$ results in the phosphorylation of Ser20 of $\mathrm{p} 53,{ }^{26}$ whereas CHK1 activation results in the phosphorylation of Ser15 of p53. ${ }^{27}$ It is clear from our results (Figures $4 d$ and e) that upon downregulation of CDK1, p53 stabilisation in response to DNA damage is achieved mostly via activation of CHK1 and not CHK2 because only the phosphorylated Ser15 of p53 is increased in response to DNA damage.

Studies performed in murine ESC have shown that complete absence of Chk2 leads to an inability to maintain G2 arrest after IR-induced damage. ${ }^{28}$ In addition, Chk2 has been reported to localise aberrantly to the centrosomes in mouse ESC and failed to translocate to the nucleus after irradiation. ${ }^{29}$ In contrast, hESC are able to activate ATMCHK2-p53 checkpoint signalling resulting in G2 arrest after administration of IR of the same dose. ${ }^{30}$ To investigate whether this G2 arrest is maintained in hESC treated with CDK1 siRNAs, we repeat cell cycle analysis after IR administration. This analysis showed that of hESC treated with control siRNA accumulate in G2/M, corroborating published data; ${ }^{30}$ however, this is compromised in the CDK1 siRNA group where a lower percentage of cells are found in the G2/M part of the cell cycle after IR (Figure 4f). It is interesting to note that the reduction of cells in the G2/M phase in the CDK1 siRNA group is accompanied by an increase in G1 phase which is perhaps due to increases in p21 and p27 expression in the CDK1-siRNA-treated groups under normal and IR conditions (Figure 4e). Unlike murine ESCs, hESCs have been shown to be capable of executing G1/S checkpoint activation in response to DNA damage. ${ }^{3,31}$ Hence, a mixed G1 and G2 arrest may be the response of hESC to protect their genome under conditions (such as CDK1 knockdown) where a full $\mathrm{G} 2 / \mathrm{M}$ arrest cannot be guaranteed.

An important role for CDK1 in mitosis progression. Given the role of CDK1/CYCLIN B1 complex in mitosis progression, it is important to investigate whether cells with reduced levels of CDK1 expression and correspondingly greater numbers of DSBs can pass through mitosis, as this would lead to propagation of DNA damage to daughter cells. Site-specific phosphorylation of histone H3 at Ser10 initiates during G2, peaks during metaphase and diminishes during late anaphase and early telophase. ${ }^{32}$ Immunofluorescence analyses (Figures 5a and c) indicated a significant increase in the number of cells expressing phospho H3 (Ser10) in the CDK1 knockdown group (Figure $5 \mathrm{~d}$ ). These results were also corroborated by western blotting (Figure $5 \mathrm{e}$ ). When this information was analysed in context of G2/M progression, it was evident that about half of the phospho $\mathrm{H} 3+$ cells from the CDK1 knockdown group accumulated at G2/prophase and the rest in metaphase/anaphase (Figure 5d). Although accumulation at G2/ prophase would enable cells to check their DNA damage and respond, the progression to metaphase and anaphase is disconcerting and suggests that these cells can escape from proper checkpoint control and progress through mitosis whilst still carrying unrepaired DSBs.

CDK1 controls many aspects of mitotic chromosome behaviour, kinetochore function and spindle microtubule dynamics to ensure accurate chromosome segregation. ${ }^{33,34}$ The presence of kinetochores is essential for proper chromosome segregation, because chromosome fragments that lack a kinetochore are not inherited faithfully and are quickly lost. ${ }^{35}$ Defects in kinetochore, in cohesion or in any of the factors that promote biorientation lead to chromosome missegregations and hence aneuploidy. ${ }^{35}$ We frequently observed abnormal mitosis, with chromosome missegregations, misaligned chromosomes and chromosome loss in the CDK1 knockdown group (Figure $5 \mathrm{c}$ ) and went on to investigate this further using immunocytochemistry with an antibody against the kinetochore-specific marker CREST. We observed proper bipolar organisation and correct numbers of kinetochores in $35.6 \%$ cells of control group; however, only $15.6 \%$ of the cells in CDK1 siRNA group were characterized by a normal kinetochore number (for an example, see Figure 6A, panel c).

Another important event for mitosis progression is the proper function and movement of centrosomes. We performed 
a

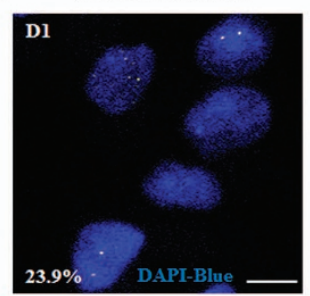

b

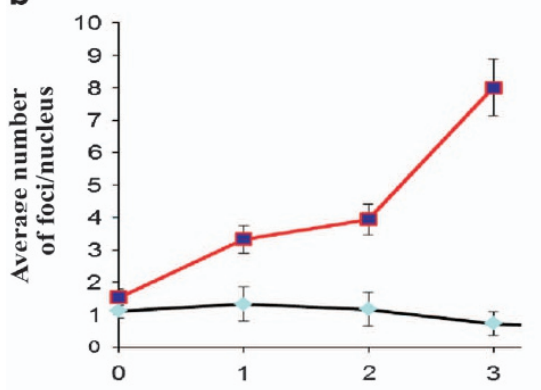

Time post transfection of siRNAs (days)
CDK1 SiRNA
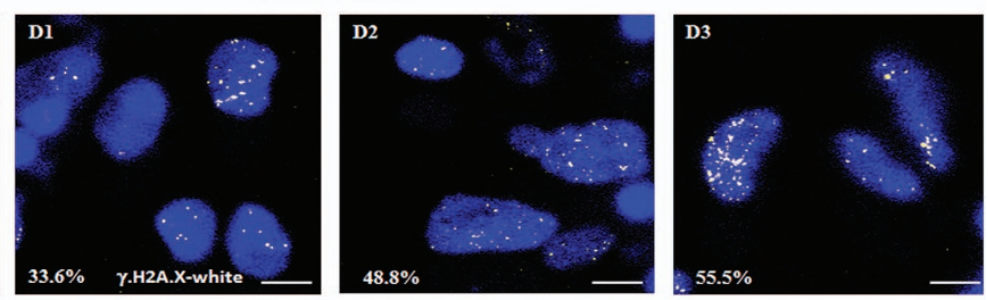

C

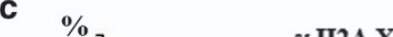

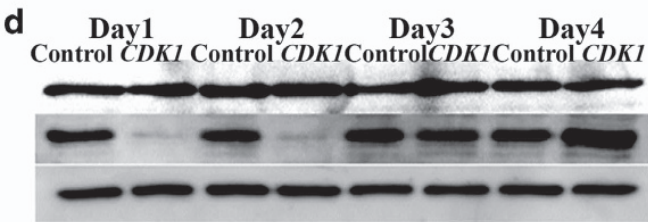

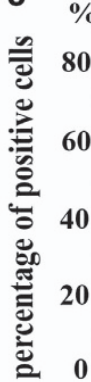

0

e Control Control IR CDK1 CDK1IR
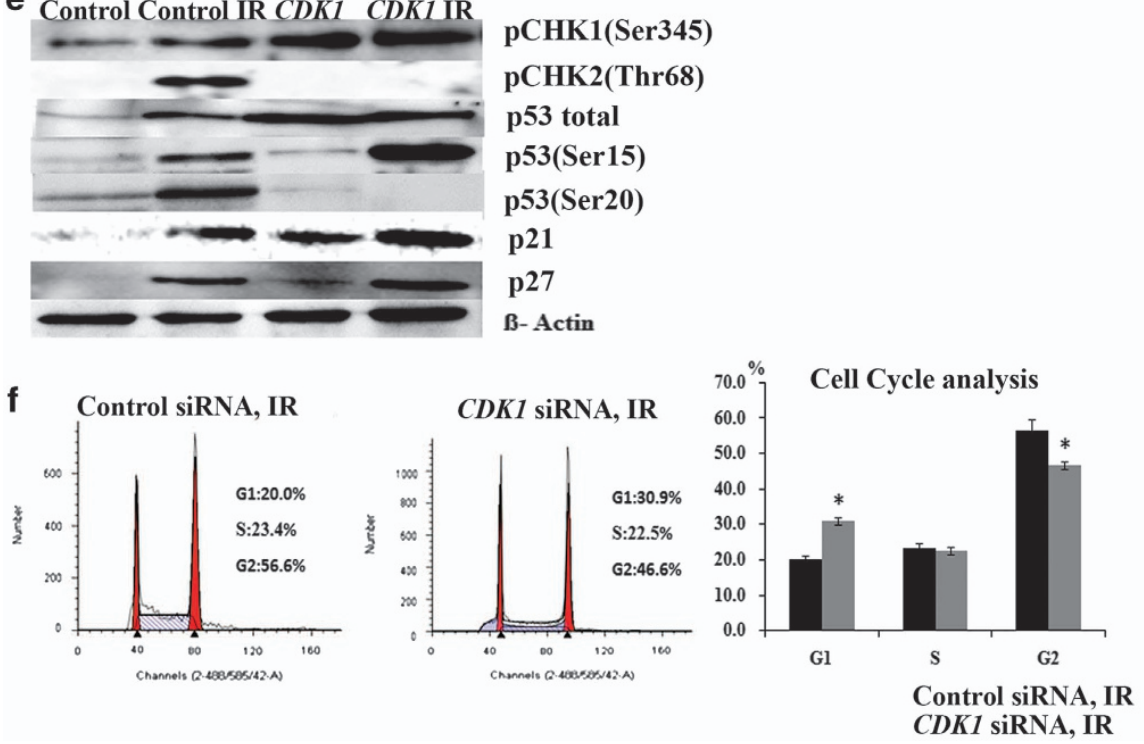

Figure 4 Knockdown of $\mathrm{CDK} 1$ induces the activation of $\gamma \mathrm{H} 2 . \mathrm{AX}$ and downregulation of $\mathrm{CHK} 2$ expression in $\mathrm{hESC}$. (a) Confocal microscopy analysis showing the presence of $\gamma \mathrm{H} 2 \mathrm{~A}$.X foci in hESC at 1,2 and 3 days post transfection of control and CDK1 siRNAs. Phosphorylated histone H2A.X ( $\gamma-\mathrm{H} 2 \mathrm{~A}$.X foci) is shown as white dots. Chromatin is stained with DAPI (blue). Scale bar $=10 \mu \mathrm{m}, \mathrm{D}=$ day. Images are representative of at least three independent experiments. Percentage of $\gamma$-H2A.X-positive cells is shown at the bottom. (b) Graphic representation of the average number of $\gamma \mathrm{H} 2 \mathrm{~A}$.X foci per nucleus in $\mathrm{hESC}$ during 3-day time course post CDK1 and control siRNAs transfections. Data areshown as average \pm S.E.M., $n=3$. (c) Flow cytometric analysis of $\gamma$-H2A.X on the second day post transfection and $16 \mathrm{~h}$ after administration of IR. Data are shown as average \pm S.E.M., $n=3$. (d) Representative images of four repeats of western blot analysis for CHK1 and CHK2 expression up to $96 \mathrm{~h}$ post transfection of siRNAS. $\beta$-Actin was used as the loading control. (e) Impacts of CDK1 inhibition on the regulation of key factors involved in $\mathrm{G} 2$ checkpoint activation analysed by western blotting at day 2 post transfection and after $6 \mathrm{~h}$ post IR on the same day (shown in the figure as IR group). $\beta$-Actin served as the loading control. The data represent at least three independent experiments. (f) Representative flow cytometric histograms at 2 days post transfection $+6 \mathrm{~h}$ post IR. The percentage of cells in each stage of cell cycle was calculated using ModFit. Graphic representation of these data is shown on the right hand panel. Results are presented as mean \pm S.E.M. $(n=3)$. T-test analysis was carried out to assess the differences in gene expression between the control and CDK1 siRNA group, ${ }^{*} P<0.05$ 

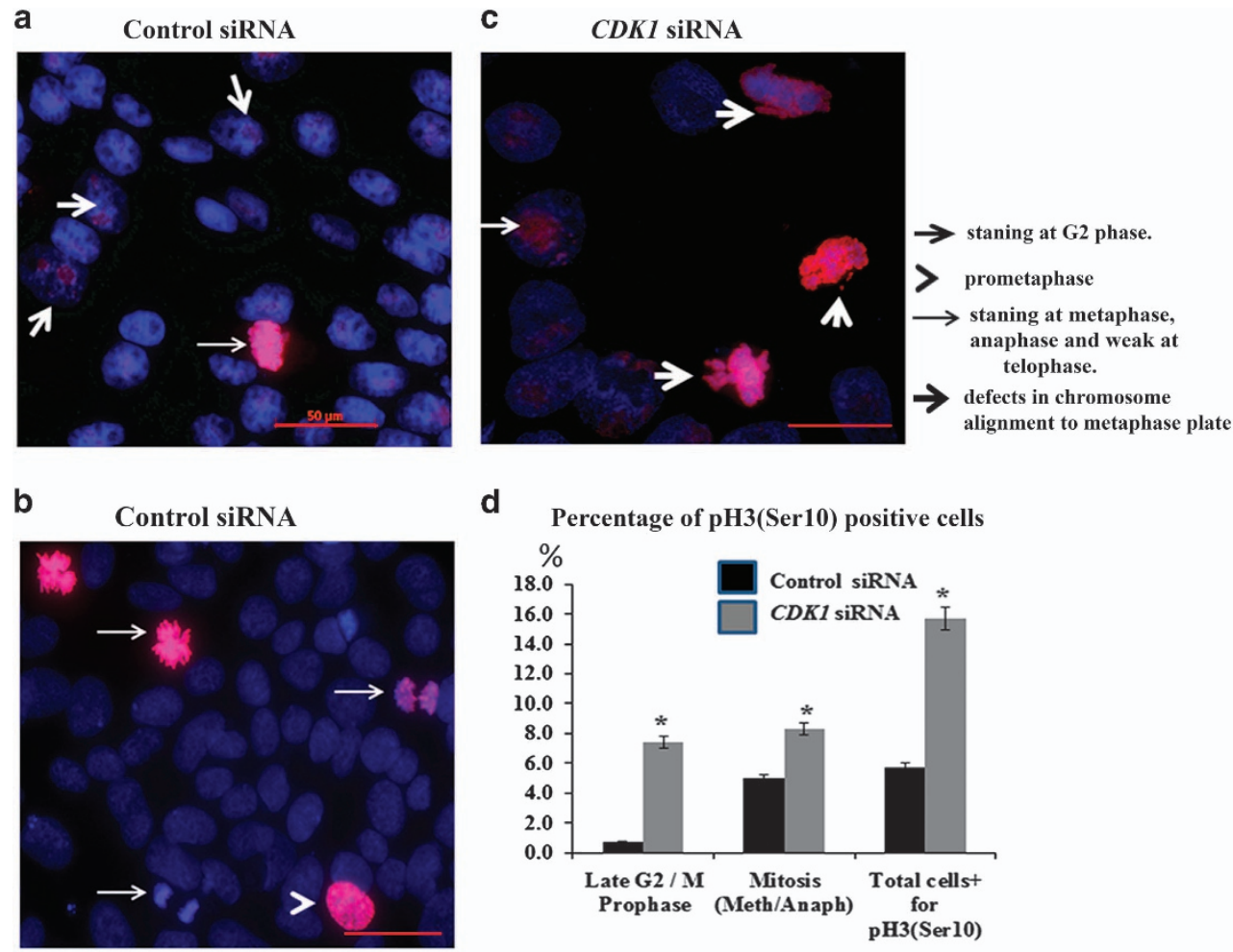

d

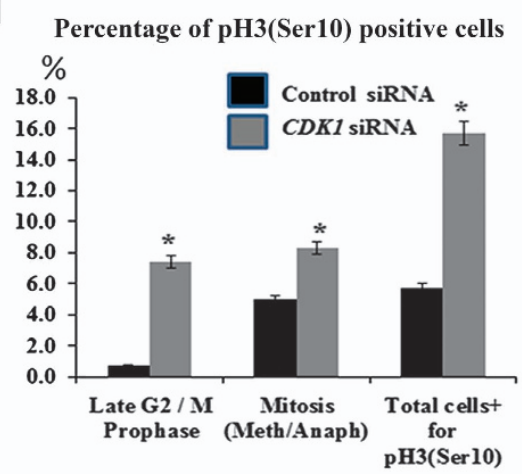

e

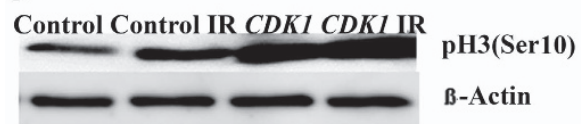

Figure 5 CDK1 is important for mitotic progression in hESC. (a-c) Representative images of immunofluorescence staining of mitotic marker pH3 Ser10 (red) at 2 days post transfection with control and CDK1 siRNAs. DNA was counterstained with DAPI (blue). Scale bar $=50 \mu \mathrm{m}$; (d) Quantification of mitotic index calculated as percentage of cells positive for phospho-Histone 3 Ser $10(\mathrm{pH} 3$ Ser10) during mitosis progression at 2 days post transfection with control and CDK1 siRNAs. The data represent mean \pm S.E.M. from three independent experiments. At least 100 cells were analysed per condition in each experiment. Statistical significance was determined by $t$ test, ${ }^{*} P \leq 0.05$. (e) Western blot analysis for $\mathrm{pH} 3 \mathrm{Ser} 10$ expression at 2 days post transfection as well as 2 days post transfection $+6 \mathrm{~h}$ post IR (shown as the IR group). $\beta$-Actin was used as the loading control. The data shown are representative of at least three independent western blots

direct visualisation of centrosomes using a PERICENTRINspecific antibody (Figure $6 \mathrm{~B}$ ). In agreement with this previous publication, we observed normal $(75 \%$ of cells in the control group and $47.3 \%$ of cells in CDK1 siRNA group; for an example see Figure 6B, panel b) and increased numbers of centrosomes (25\% of cells in control group and $52.7 \%$ of cells in the CDK1 siRNA group (Figure 6B, panels: $\mathrm{a}, \mathrm{c}, \mathrm{g}, \mathrm{h}$ ) both in the control and in CDK1 knockdown groups. Nevertheless, the percentage of cells with increased centrosome number in the CDK1 siRNA group suggests an altered centrosome biogenesis upon CDK1 downregulation, which could be due to an inability to activate $\mathrm{CHK} 2$ expression in response to DNA damage. In support, it has been shown that $\mathrm{CHK} 2$ localises at centrosomes during mitosis and alteration of $\mathrm{CHK} 2$ function promotes chromosome segregation errors in dividing cells, a feature which is commonly observed in cancer cells and might drive chromosomal instability and cellular transformation. ${ }^{36}$

Centriole function, duplication and organisation are important events for mitotic progression. ${ }^{37,38}$ Immunofluorescence analysis with a centriole-specific marker, CENTRIN-2, also revealed defects in the distribution and number of this organelle upon $C D K 1$ downregulation (Figure $6 \mathrm{C}$, panels $\mathrm{d}-\mathrm{k}$ ).
Although normal CENTRIN-2 staining was observed in $82.7 \%$ of control cells (for an example, see Figure 6C, panel a), only $41.5 \%$ of the cells in the CDK1 siRNA group showed normal centriole numbers. Together these data suggest that CDK1 downregulation contributes to abnormal kinetochore, centrosome and centriole biogenesis and that all these events could be the causative factor leading to the observed chromosomal abnormalities in the CDK1 knockdown group (Supplementary Figure 4).

CDK1 downregulation leads to PARP1 activation but impaired apoptosis in hESC. The CDK1/CYCLIN B1 complex is able to interact and phosphorylate both pro- and anti-apoptotic proteins such as Bad, Caspase 9, Caspase 8, Caspase 2, Caspase3, Bcl-2, Bcl-xl, Mcl-1 and Survivin. ${ }^{39-43}$ We performed western blotting analysis and observed that the expression of anti-apoptotic/survival proteins (such as Survivin, BCL2, BCL-xL) was downregulated in the CDK1 siRNA group (Figure 7a). In addition, the expression of proapoptotic protein BAD was increased upon CDK1 downregulation. At the same time, the expression of active $B A X$ (pro-apoptotic protein) was downregulated (Figure 7a); 
however, this is more likely to reflect the loss of pluripotency in hESC upon CDK1 downregulation, as it has recently been shown that active BAX is only detected in undifferentiated hESC. ${ }^{44}$ We also performed flow cytometric analysis for expression of CASPASE 3 and 9, because it has been shown that the loss of phosphorylation on Thr34 of SURVIVIN can result in the disassociation of the Survivin-Caspase 9 complex and initiation of caspase 9-dependent apoptosis;
A

Control siRNA

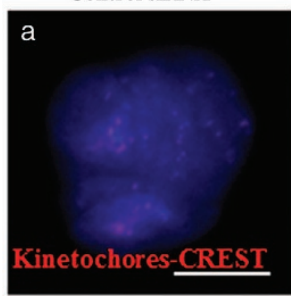

B

Control siRNA

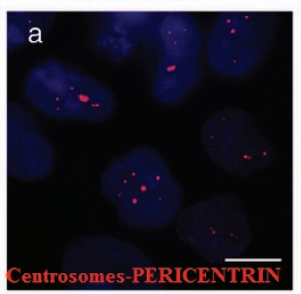

CDK1 SIRNA

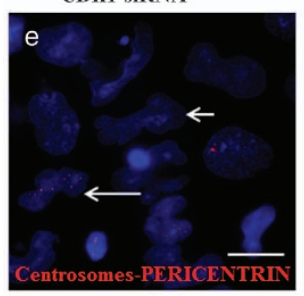

C

Control siRNA

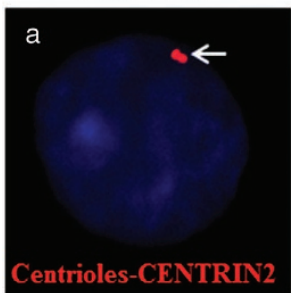

CDK1 SiRNA
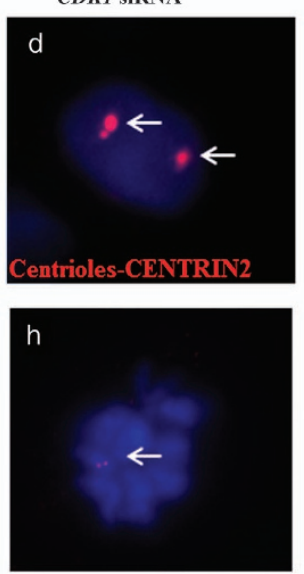

Kinetochores-CREST

CDKI SIRNA

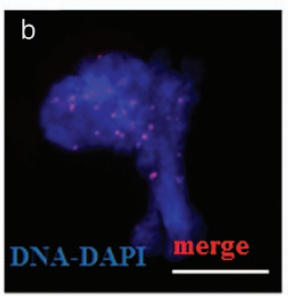

Centrosomes- PERICENTRIN
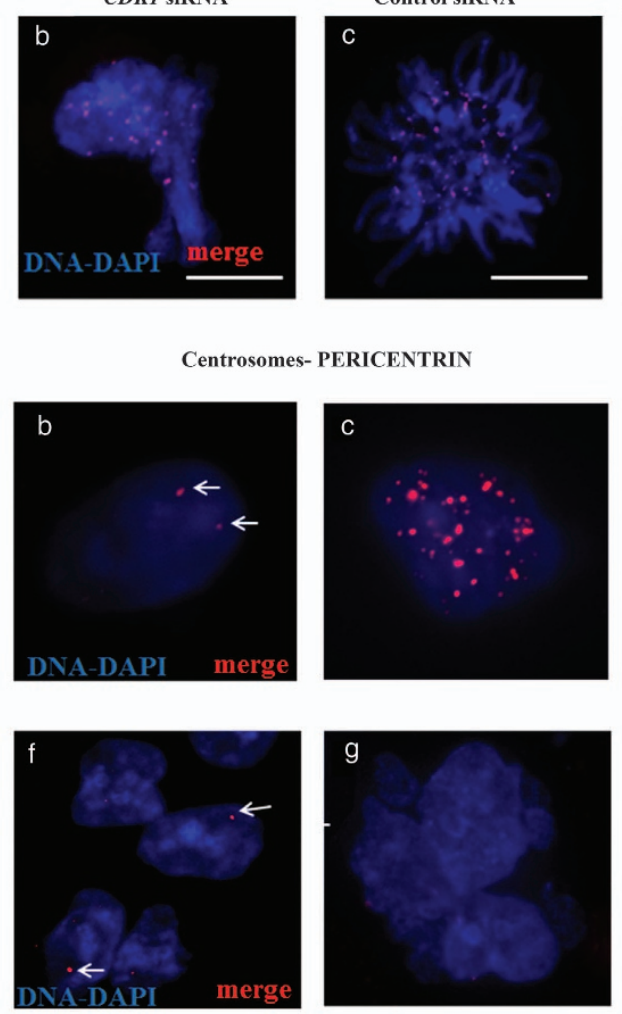

Centrioles-CENTRIN2
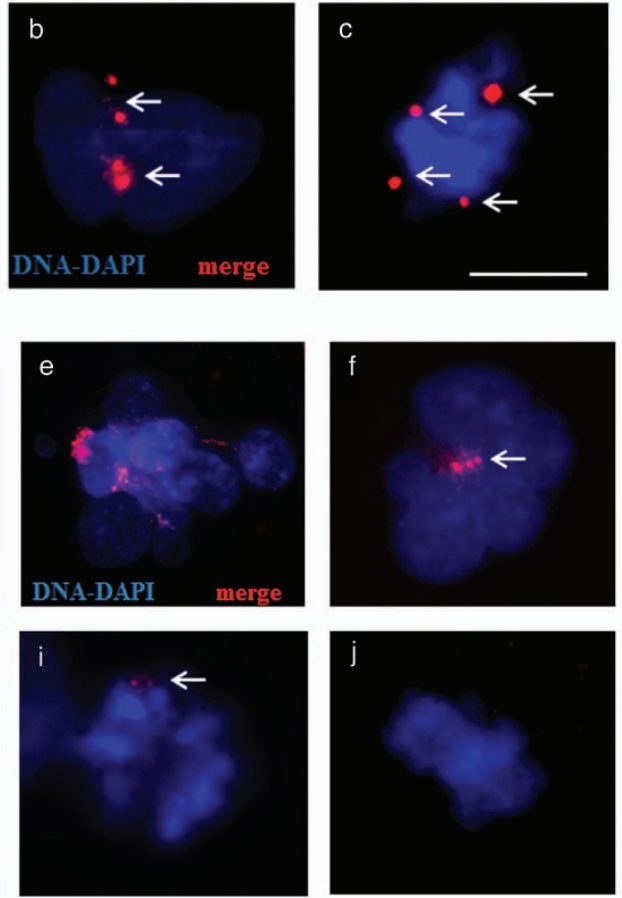
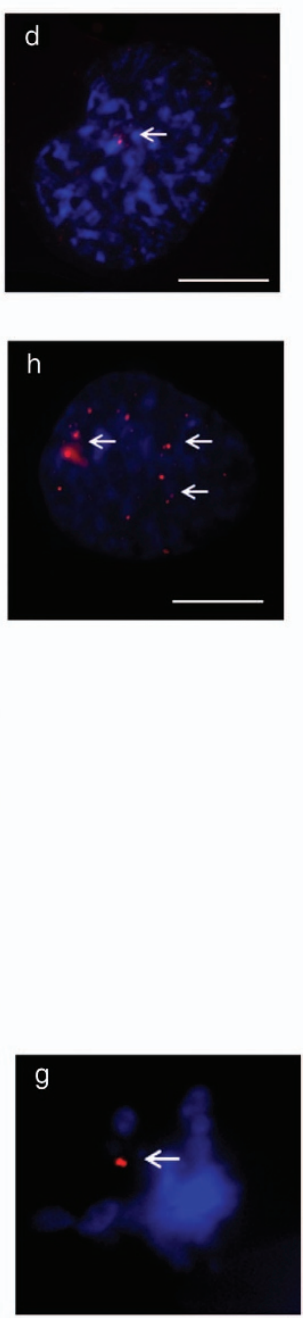

CDK1 SIRNA
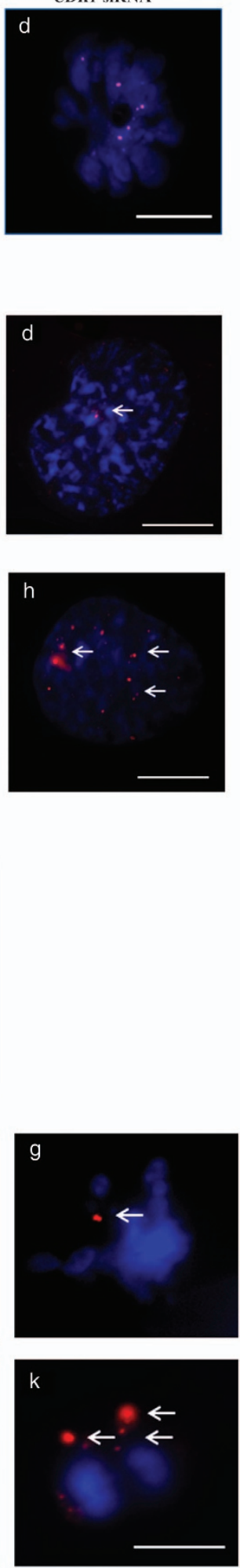
however, we were unable to detect significant changes between the control and CDK1 siRNA groups (Figure 7b). Another important apoptosis executor is PARP1 (poly ADP ribose polymerase 1 ), whose activity increases substantially in response to cellular stress. ${ }^{45}$ Flow cytometric analysis indicated significant upregulation of active PARP1 upon CDK1 downregulation (Figure 7c); however, TUNEL analysis did not reveal significant differences in the number of apoptotic cells, suggesting an inability to commit to apoptosis in the absence of CDK1 (Figure 7d). Similar data were obtained in hiPSCs (Supplementary Figure 5).

However, upon increased DNA damage (such as IR), commitment to apoptosis occurs (Figure 7d), suggesting that the threshold of accumulated DNA damage is the factor behind the hESC decision to expunge damaged cells either through induction of differentiation (steady state culture conditions) or apoptosis (IR conditions).

\section{Discussion}

Our results suggest that downregulation of $C D K 1$ leads to the loss of hESC and hiPSC pluripotency and upregulation of a variety of lineage markers that are not only associated with differentiation to trophoectoderm but also extend to other lineages such as ectoderm, mesoderm and endoderm. That led us to speculate that the impacts of $C D K 1$ downregulation must go beyond a single interaction with OCT4. ${ }^{17}$ With this in mind, we screened the list of published target genes that are transcriptionally activated by pluripotency factors and also the range of substrates that are phosphorylated by CDK1 in hESC. ${ }^{20,46}$ We found that $C D K 1$ is transcriptionally regulated by SOX2 and NANOG. ${ }^{20,46}$ Furthermore, a very recent paper that was published whilst this manuscript was under review showed that NANOG, a key pluripotency factor, is directly phosphorylated by CDK1. ${ }^{47}$ This later direct interaction has not been explored functionally; hence, it is impossible to conclude whether the impacts of CDK1 on maintenance of pluripotency are through direct interactions with pluripotency factors or indirectly through the large number of targets it may phosphorylate on human pluripotent stem cells.

Associated to these published data are also our important findings of polyploidy occurrence ( $14 \%$ of cell population) and multiple chromosomal abnormalities upon CDK1 downregulation. For genomic instability to occur, there must be problems with the cell's ability to activate checkpoint signalling and repair DNA damage, its inability to activate and/or execute apoptosis and/or cell intrinsic problems during chromosome separation/cell division. To investigate which of these scenarios is prevalent when $C D K 1$ is downregulated, we first assessed how hESCs protect their genomes against DNA damage. We found that cells with reduced CDK1 expression show almost three times as much accumulation of unrepaired DSBs compared with the control group, suggesting either a higher generation of DSBs or/and impaired ability to repair DSBs. Currently, it is unclear whether this is due to increased generation of DSBs during DNA replication or an inability to repair them via $\mathrm{HR}$ as $\mathrm{CDK} 1$ has been known to interact closely with the replication machinery as well as DSB DNA repair systems. Our data showing a very high percentage of cells with DSB foci in the $S$ phase of the cell cycle are supportive of these two hypothesis; however, further work is needed to investigate this. Furthermore, upon exposure to IR, those cells are unable to maintain the G2/M arrest that is typical for hESC. ${ }^{30}$ Instead, a mixture of G1 and G2/M arrest was noticed for a prolonged time after administration of IR, leading us to investigate further the pattern of $\mathrm{CHK} 1$ and CHK2 activation upon CDK1 downregulation. We noticed that whilst CHK1 activation and downstream p53-dependent transactivation was occurring, $\mathrm{CHK} 2$ activation was completely impaired. Although activation of CHK1 can compensate for some of the CHK2 functions, it is clear from our results that this is not sufficient to maintain a fully functional G2/M checkpoint arrest in response to DNA damage and to repair the DSBs arising under normal culture and stress conditions, when $C D K 1$ is downregulated in hESC.

Given the improper maintenance of G2/M checkpoint in the CDK1 siRNA group, we went on to investigate mitotic progression using the mitosis-specific marker, phosphoHistone 3 (Ser10). We observed that a significantly higher number $(15.7 \%)$ of cells in the CDK1 siRNA group were at mitosis when compared with the control group (6\%), suggesting an inappropriate escape from the G2 checkpoint back into the cell cycle, likely to result in tolerance to cells bearing DNA damage and/or unstable genomes. We analysed kinetochore, centrosome and centriole numbers and in all cases, we found a higher percentage of cells with altered distribution and number of these organelles. These organelles are essential for proper spindle microtubule formation, chromosome segregation and cell division; hence, the presence of multiple abnormalities of these organelles could lead to the formation of dicentric chromosomes as observed in the CDK1 siRNA group. In addition to chromosomal abnormalities, CDK1 downregulation also led to polyploidy in about $14 \%$ of the cell population corroborating previous data on somatic cells ${ }^{48-51}$ and suggesting an important role for CDK1 in full and proper execution of mitosis and cytokinesis.

In the absence of proper DNA damage repair and presence of mitotic deficiencies, execution of apoptosis for

Figure 6 Downregulation of CDK1 leads to multiple abnormalities in kinetochore, centrosome and centriole numbers in hESC. (A) Specific antibody to kinetochores, CREST (red), was used for immunofluorescence staining of hESC treated with control siRNA $(\mathbf{a}, \mathbf{c})$ and CDK1 siRNAs $(\mathbf{b}, \mathbf{d})$ at 2 days post transfection. DNA was counterstained with DAPI (blue) in all images. Examples of hESC with supernumericial kinetochores at interphase nuclei observed in control siRNA (a) and CDK1 siRNA-treated cells (b). (B) Immunostaining of centrosomes with a specific antibody, Pericentrin (red). Examples of $h E S C$ with normal $(\mathbf{b}, \mathbf{d}, \mathbf{f})$ and supernumerary centrosomes (a, $\mathbf{c}, \mathbf{g}, \mathbf{h})$ at 2 days post transfection with control and CDK1 siRNAs, respectively. Note abnormal nucleus blebbing on the CDK1 siRNA group shown by long arrows (e). Centrosomes are shown by short white arrows. (C) Centrin2-specific antibody (red) for visualisation by immunofluorescence of centrioles at 2 days post $h E S C$ transfection with control siRNA (a, b, c) and CDK1 RNAi (d-k). Normal (a: two centrioles; pointed by arrow) and abnormal centriole number (b) was observed in the control siRNA-transfected group in interphase nucleuses (b) and during mitosis (c). White arrows point to centrioles. Knockdown of CDK1 in hESC causes abnormalities in centriole number in interphase nuclei (d), nuclei blebbing with abnormal centriole number $(\mathbf{e}, \mathbf{f})$ and abnormal mitoses $(\mathbf{g}-\mathbf{k})$ with wrong distribution and number of centrioles $(\mathbf{g}-\mathbf{k})$. A-C: Scale bar $=5 \mu \mathrm{m}$. Images are representative of at least three independent experiments 
a

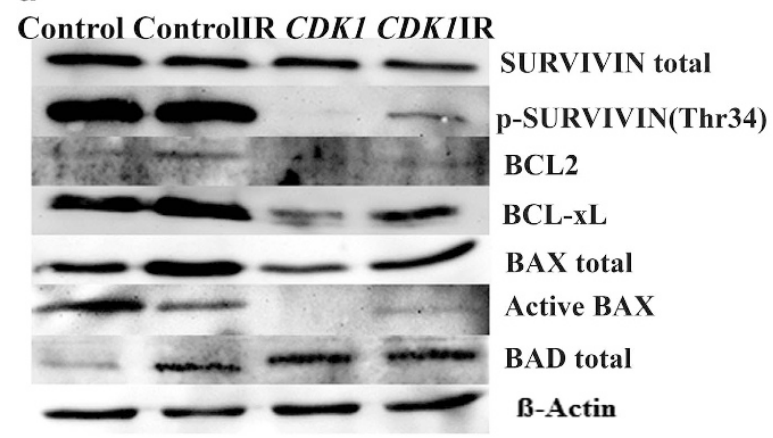

\section{b}

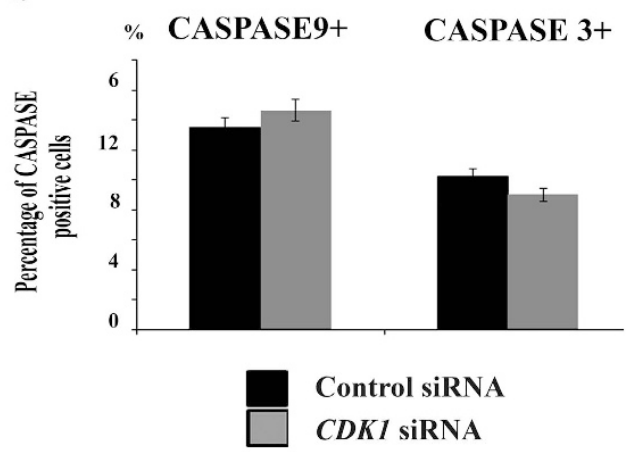

。

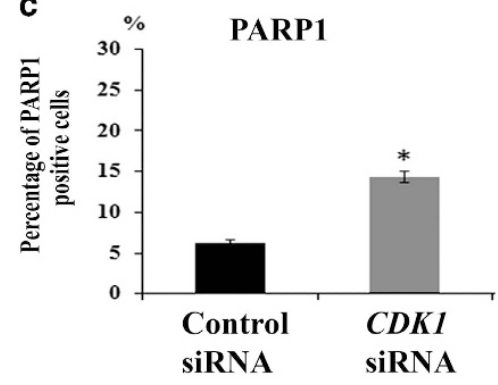

d

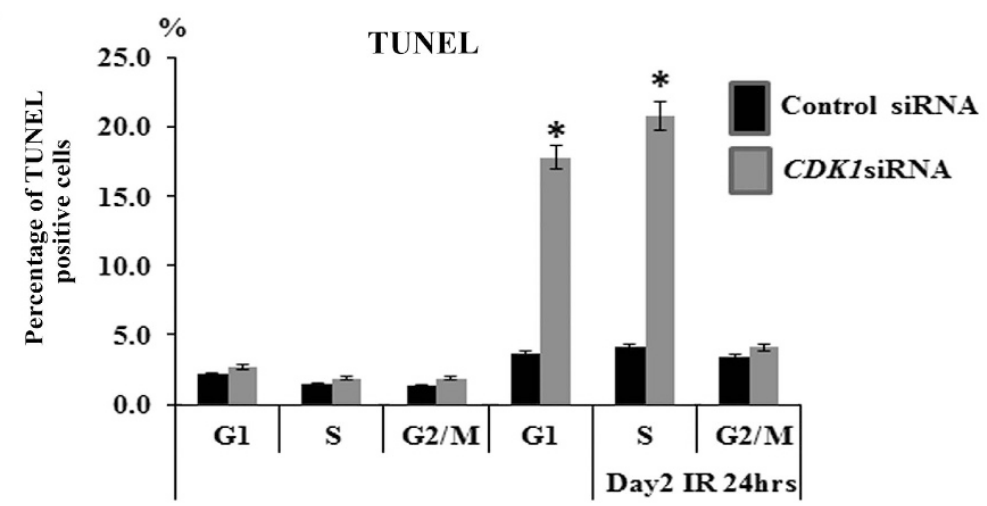

Figure 7 Knockdown of CDK1 in hESC results in PARP1 activation, but impaired apoptosis. (a) Western blot analysis showing downregulation of pro-survival proteins and activation of pro-apoptotic markers at 2 days post transfection of $\mathrm{hESC}$ with control and CDK1 siRNAs in the absence and presence of IR (extracts were collected $16 \mathrm{~h}$ post IR). Images are representative of at least three independent experiments. $\beta$-Actin served as the loading control. (b) Graphical representation of flow cytometric analysis for caspase 9 and 3 activation in hESC at 2 days post transfection with control and CDK1 siRNAs. Results are presented as mean \pm S.E.M. $(n=3)$. (c) Graphical representation of flow cytometric analysis for PARP1 activation in hESC at 2 days post transfection with control and CDK1 siRNAs. Results are presented as mean \pm S.E.M. ( $n=3)$. T-test analysis was carried out to assess the differences in gene expression between the control and CDK1 siRNA group. (d) Graphical representation of TUNEL analysis at each stage of cell cycle at 2 days post transfection with control and CDK1 siRNA under normal culture conditions as well as IR. Results are presented as mean \pm S.E.M. ( $n=3)$, $t$-test analysis was carried out to assess differences in gene expression between the control and CDK1 siRNA group, ${ }^{*} P<0.05$

elimination of damaged cells with genomic instability becomes very important. Indeed, it has been suggested that $\mathrm{hESC}$ s prefer to eliminate damaged cells by apoptosis rather than undergo DNA repair. ${ }^{52}$ This was not the case as there was no significant increase in activation of CASPASE 3 and 9 in the $C D K 1$ downregulation group when compared with the control. We then investigated the activity of PARP-1 which is known to trigger the release of the mitochondrial apoptosis-inducing factor that promotes programmed cell death through a caspase-independent pathway. ${ }^{53}$ Although PARP1 activity was increased upon CDK1 downregulation in hESC, we failed to detect a significant commitment to apoptosis in both hESC and hiPSC; however, administration of IR (which is known to cause increased DSBs) does result in enhanced apoptosis in hESC deficient for CDK1. Together, these data suggest that commitment to apoptosis in hESC and hiPSC is impaired in the absence of CDK1; however, this is dependent on the level of accumulated DNA damage, such that above a certain threshold, apoptosis is preferred rather than stem cell differentiation. Hence, loss of pluripotency and induction of differentiated state in the CDK1 siRNA group could be the pluripotent stem cells response to restore the genomic stability by coupling the mitotic checkpoint control to execution of apoptosis upon induction of differentiation (refer to Figure 8 for a schematic summary). 
Materials and Methods

Pluripotent stem cell culture and transfection experiments. The human $\mathrm{H} 9$ embryonic stem cell line (WiCell Research Institute, Madison, MI, USA) line was cultured on mitotically inactivated mouse embryonic fibroblasts and passaged as described by Neganova et al. ${ }^{2}$ Human iPSC (SB-NEO1) was generated from reprogramming of neonatal fibroblasts using the Sendai-based Cytotune 1 kit provided by Life Technologies (Paisley, UK). Full in vitro and in vivo characterisation was performed as part of the STEMBANCC European project and can be found in the StemBio Gateway. Human iPSCs were cultured the same way as hESCs on mitotically inactivated murine embryonic fibroblasts. A few passages prior to start of experiments, hESCs and hiPSCs were transferred to Matrigelcoated plates with feeder-conditioned media as previously described Neganova et al. ${ }^{2}$ Downregulation of CDK1 was achieved using siRNAs: CDK1 siRNAs Validated Stealth RNAi DuoPak [CDC2VHS40172; duplex 1: (RNA) - CCU AGU ACU GCA AUU CGG GAA AUU $U$ and duplex 2: (RNA) - GGA CAA UCA GAU UAA GAA GAU GUA G] from (Invitrogen Ltd, Paisley, UK; www. invitrogen.com) following the protocol reported in our previous publication. ${ }^{2}$ The cells were analysed at 24, 48, 72 and $96 \mathrm{~h}$ after transfection. Cell synchronisation at particular stages of the cell cycle was performed as described before. ${ }^{2}$

Karyotype analysis. Karyotypes were determined by Standard G-Banding Procedure. At least 30 metaphases were analysed for each experiment.

Western immunoblotting and immunoprecipitation. Protein extraction, western blotting and immunoprecipitation were performed as described in our previous publication. ${ }^{2}$ Primary antibodies used in this work were purchased from Santa Cruz Biotechnology Ltd (Middlesex, UK) and Cell Signalling (Danvers, MA, USA): Cdc2 p34; CDK2; SURVIVIN; p-SURVIVIN (Thr34); cyclin B1; p-Histone H3 (Ser10); p-53; p-Chk1 (Ser345); p-Chk2 (Thr68); Chk1; Chk2; Cyclin A, p21, p27 and $\beta$-Actin. Bcl-xL; Bcl-2, BAX, active BAX; BAD; Cdc2; p-cdc2 (Tyr15); $\mathrm{p}$-Cdc2 (Thr161); p-Cdc2 (Tyr15/Thr14); p-p53(Ser15) and p-p53(Ser20). The antibodies to $\beta$-actin and/or GAPDH (Abcam) were used after membrane stripping to confirm uniform protein loading. Antibody/antigen complexes were detected using ECL (Amersham Biosciences, Little Chalfont, UK; www.gelifesciences.com) and images were acquired using a luminescent image analyser FUJIFILM and LAS-3000 software (FUJl, Abingdon, UK; www.rndsystems.com).

Quantitative reverse transcription-polymerase chain reaction. Total RNA was extracted using TRIzol reagent (Invitrogen) according to the manufacturer's instructions. Following DNase treatment using RQ1 DNasel (Promega, Mannheim, Germany; http://www.promega.com), cDNA was synthesized using SuperScript Reverse Transcriptase (Invitrogen). qRT-PCR analysis was

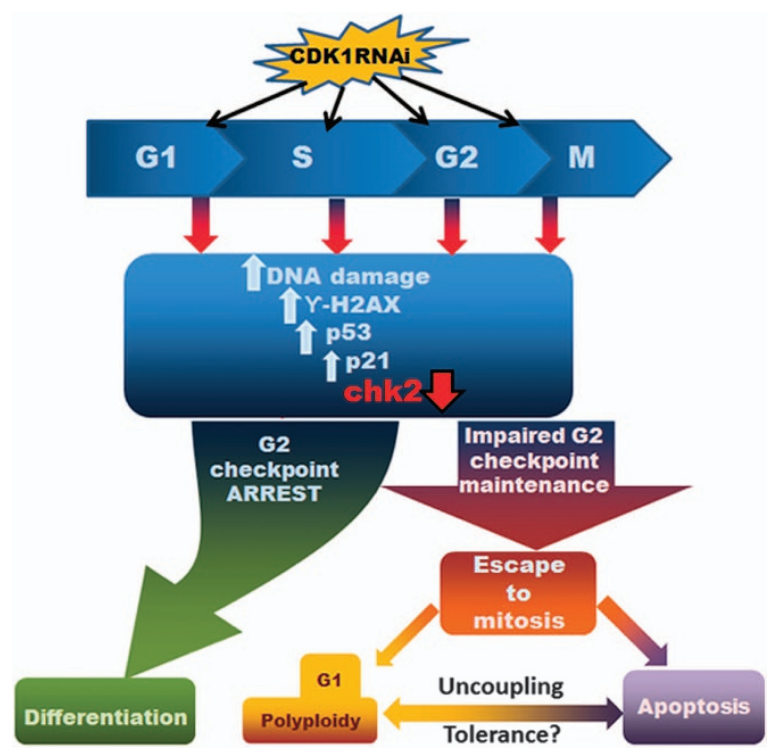

Figure 8 A schematic presentation of CDK1's function in maintenance of pluripotency and genomic stability in human pluripotent stem cells carried out using SYBR Green PCR master mix (Sigma Aldrich, Dorset, UK) and the primers are listed in supporting information Table 1. All samples were analyzed using an AB7900HT real-time analyzer (Life Technologies) and were normalized to GAPDH, RPL13A and SDHA expression.

Cell cycle analysis. hESCs and hiPSCs were collected using Accutase (Chemicon, Temecula, CA, USA; www.millipore.com). Cell cycle analysis was performed using the CycleTest Plus DNA reagent kit (BD Biosciences, Oxford, UK) using a FACS Canto (BD Biosciences). The data were analysed using ModFit (Verity software House, Topsham, ME, USA; www.vsh.com) to generate percentages of cells in G1, S and G2/M phases. At least 10000 cells were analysed in each experiment.

Immunocytochemistry and confocal microscopy. Briefly, hESCs were cultured on Matrigel-covered glass slide flasks (SlideFlask, NUNC, Roskilde, Denmark; www.nuncbrand.co) in the presence of feeder-conditioned media. Cells were quickly washed with phosphate-buffered saline (PBS), prior to being fixed with $2 \%$ formaldehyde for 10 min and permeabilized with $0.1 \%$ Triton X-100 in PBS for $15 \mathrm{~min}$ at room temperature. Unspecific binding was blocked by incubation of samples in PBS containing $5 \%$ normal goat serum for $40 \mathrm{~min}$. Staining with the mouse monoclonal anti-phospho-histone H2A.X (Ser 139; Millipore, Watford, UK; www.millipore.com) was carried out as described before. ${ }^{2}$ Slides were examined using a Zeiss confocal microscope (Carl Zeiss, Jena, Germany; http://www.zeiss. com). Quantification was performed by counting -H2A.X-positive foci in 150-200 nuclei per experiment.

For other immunocytochemical analyses, the cells were fixed in $4 \%$ (wt/vol) paraformaldehyde for $20 \mathrm{~min}$. An additional permeabilization step in $0.2 \%$ (vol/vol) Triton X-100 in PBS was performed prior to staining with primary antibodies. Blocking step was performed by incubation in $1 \%$ (wt/vol) bovine serum albumin or alternatively in $10 \%$ (vol/vol) goat serum. Cells were incubated with primary antibodies overnight at $4{ }^{\circ} \mathrm{C}$ and secondary antibodies for $2 \mathrm{~h}$. Primary antibodies used in this study are anti-Ki67, anti-Cdc2, anti-Cyclin B1, anti- Cyclin A and anti-pHistone H3 (Ser10), all purchased from Santa Cruz Biotechnology. The nuclei were counterstained with DAPI. The bright-field and fluorescent images were obtained

Table 1 The sequences of oligonucleotides used for qRT-PCR analysis.

\begin{tabular}{|c|c|}
\hline Gene Name & Primer (5'-3') \\
\hline$C D K 1$ & $\begin{array}{l}\text { For: TTTTCAGAGCTTTGGGCACT } \\
\text { Rev: CCATTTTGCCAGAAATTCGT }\end{array}$ \\
\hline OCT4 & $\begin{array}{l}\text { For: AGCTCTGCAGAAAGAGTCCCAGG } \\
\text { Rev: TGAGCCCCACATCGGCCTGT }\end{array}$ \\
\hline$K L F 4$ & $\begin{array}{l}\text { For: CCCAATTACCCATCCTTCCT } \\
\text { Rev: CGTCCCAGTCACAGTGGTAA }\end{array}$ \\
\hline Lin28 & $\begin{array}{l}\text { For: TCCTGCACTGTGTTCTCAGG } \\
\text { Rev: AAAGCCAGCTCTTATTGGCA }\end{array}$ \\
\hline SOX2 & $\begin{array}{l}\text { For: GGCAGCTACAGCATGATGCAGGACC } \\
\text { Rev: CTGGTCATGGAGTTGTACTGCAGG }\end{array}$ \\
\hline NANOG & $\begin{array}{l}\text { For: TCCAGCTTGTCCCCAAAGCTTGC } \\
\text { Rev: ACAGTCTCCGTGTGAGGCATCT }\end{array}$ \\
\hline$C D \times 2$ & $\begin{array}{l}\text { For: GGCAGCCAAGTGAAAACCAG } \\
\text { Rev: GGTGATGTAGCGACTGTAGTGAA }\end{array}$ \\
\hline GATA4 & $\begin{array}{l}\text { For: ACACCCCAATCTCGATATGTTTG } \\
\text { Rev: GTTGCACAGATAGTGACCCGT }\end{array}$ \\
\hline PAX6 & $\begin{array}{l}\text { For: ACAGTCACAGCGGAGTGAATC } \\
\text { Rev: ACTTTTGCATCTGCATGGGTC }\end{array}$ \\
\hline FGF5 & $\begin{array}{l}\text { For: ATTTGCTGTGTCTCAGGGGAT } \\
\text { Rev: CTGTGAACTTGGCACTTGCAT }\end{array}$ \\
\hline NESTIN & $\begin{array}{l}\text { For: CAGGAGAAACAGGGCCTACA } \\
\text { Rev: TGGGAGCAAAGATCCAAGAC }\end{array}$ \\
\hline$B R A C H Y U R Y$ & $\begin{array}{l}\text { For: TCAGCAAAGTCAAGCTCACCA } \\
\text { Rev: CCCCAACTCTCACTATGTGGATT }\end{array}$ \\
\hline$A F P$ & $\begin{array}{l}\text { For: CTTTGGGCTGCTCGCTATGA } \\
\text { Rev: ATGGCTTGGAAAGTTCGGGTC }\end{array}$ \\
\hline$G A P D H$ & $\begin{array}{l}\text { For: TGCACCACCAACTGCTTAGC } \\
\text { Rev: GGCATGGACTGTGGTCATGAG }\end{array}$ \\
\hline$R P L 12 A$ & $\begin{array}{l}\text { For: CCTGGAGGAGAAGAGGAAAGAGA } \\
\text { Rev: TTGAGGACCTCTGTGTATTTGTCAA }\end{array}$ \\
\hline$S D H A$ & $\begin{array}{l}\text { For: TGGGAACAAGAGGGCATCTG } \\
\text { Rev: CCACCACTGCATCAAATTCATG }\end{array}$ \\
\hline
\end{tabular}


using a Zeiss microscope and the AxioVision software (Carl Zeiss). At least 100 cells were analysed for each technical replicate.

TUNEL Assay. Analysis of DNA fragmentation was performed with APODIRECT kit (BD Pharmingen, Oxford, UK). Cells were prepared according to manual instructions and analysed by flow cytometry. Flow cytometric analysis (BD Biosciences LSRII) with two dyes, namely propidium iodide for staining total DNA and FITC-dUTP for staining the apoptotic cells, was carried out. At least 10000 events were analysed in each experiment.

CASPASE activity assay. APO LOGIX Carboxyfluorescein (FAM) Caspase Detection kit (Cell Technology, Kennesaw, GA, USA) was used to detect active caspases in living cells through the use of a carboxyfluorescein (FAM)-labelled peptide fluoromethyl ketone (FMK) caspase inhibitor (FAM-Peptide-FMK). The FAMpeptide inhibitor (FAM-LEHD-FMK) irreversibly binds to active caspase 9 and FAMDEVD-FMK inhibitor to active caspase 3. Flow cytometry (LSRII, BD Biosciences) was used to measure the percentage of caspase 9-positive cells. At least 10000 events were analysed in each experiment.

Alkaline phosphatase staining. AP staining was carried out using the AP Detection kit according to manufacturer's instructions (Chemicon, Temecula, CA, USA). The bright-field images were obtained using a Zeiss microscope and AxioVision software (Carl Zeiss).

Flow cytometric analysis for assessing apoptosis, DNA damage and cell proliferation. This was performed using a flow cytometric kit (cat. no. 562253; BD Biosciences) following the manufacturer's instructions. In brief, hESCs were labelled with BrdU, then fixed, permeabilized and treated with DNAse. Following this treatment, cells were simultaneously stained with PerCP-Cy5.5-labelled BrdU, PE-labelled anti-cleaved PARP and Alexa Fluor 647-labelled anti- $\gamma \mathrm{H} 2 \mathrm{AX}$. Cells were resuspended in staining buffer and analysed by flow cytometry. Cell cycle distribution was analysed by adding DAPI. At least 10000 events were recorded for each sample. Annexin-V-PE apoptosis detection kit (BD Bioscience, Oxford, UK; www. bdbiosciences.com) was also used as described before in Neganova et al. ${ }^{3}$

Statistical analysis. $T$-test analysis was used to assess differences between the control and CDK1 siRNA group. The results were considered significant if $P \leq 0.05$.

Indirect immunofluorescence for kinetochores, centrosomes and centrioles. For immunodetection of kinetochores, cells were fixed in $2 \%$ paraformaldehyde for $5 \mathrm{~min}$ at room temperature and in cold methanol for $5 \mathrm{~min}$, then washed for $5 \mathrm{~min}$ in MBST buffer containing $10 \mathrm{mM}$ 3-(N-Morpholino)- propane sulfonic acid ( $\mathrm{pH} 7.2), 150 \mathrm{mM} \mathrm{NaCl}$ and $0.05 \%$ Tween- 20 . The cells were blocked with $5 \%$ FBS in MBST buffer and incubated with human anti-centromere sera (CREST, 90C-CS-1058, Europa Bioproducts, Cambridge, UK; www.europabioproducts.com) diluted in MBST buffer containing $2 \%$ FBS. For immunodetection of pericentrin, $\mathrm{hESC}$ were fixed in an $95 \%$ ethanol/ $1 \%$ acetic acid solution for 30 min on ice, transferred into PBS and permeabilized for 15 min with $0.1 \%$ Triton-X, blocked for $1 \mathrm{~h}$ with $1 \%$ bovine serum albumin in PBS (pH 7.4) containing $0.05 \%$ Tween-20 and incubated overnight at $4^{\circ} \mathrm{C}$ with primary antibody against pericentrin (ab4448, Abcam, Cambridge, UK). For immunodetection of centrin, cells were fixed in ice-cold methanol:acetone $(9: 1)$ solution for $20 \mathrm{~min}$, washed with PBS and permeabilized for $15 \mathrm{~min}$ with $0.1 \%$ Triton-X, blocked for $1 \mathrm{~h}$ with $1 \%$ bovine serum albumin in PBS (pH 7.4) containing $0.05 \%$ Tween-20 and incubated overnight at $4{ }^{\circ} \mathrm{C}$ with anti-centrin-2 (sc-27793-R, Santa Cruz Biotechnology). Incubations with secondary antibodies were carried out for $1 \mathrm{~h}$ at room temperature. Cell nuclei were counterstained with 4', 6-diamidino-2-phenylindole (DAPI, Sigma-Aldrich). Microscopy was performed using Zeiss microscope with Z-scanned (step $0.15 \mu \mathrm{m}$ ) using $\times 63$ objective and the AxioVision software (Carl Zeiss). At least 10 fields with were analysed for each of the three technical replicates.

\section{Conflict of Interest}

The authors declare no conflict of interest.

Acknowledgements. We are grateful to Conselleria de Sanidad (Generalitat Valenciana), the Instituto de Salud Carlos III (Ministry of Science and Innovation), awards from BBSRC/EPSCR(CISBAN) and Newcastle University for providing financial support for this work. We would also like to thank Prof. Penny Jeggo for her critical assessment of the manuscript, lan Dimmick and Owen Hughes for help with the flow cytometric analysis, Lukas Cucera for help with kinetochore, centrosome and centriole number analysis, Lisa Hodgson for help with microscopy and Gavin Cuthbert for help with cytogenetic tests. We also would like to thank Mr A. Rakovschik for help with figure preparation.

1. Zhang X, Neganova I, Przyborski S, Yang C, Cooke M, Atkinson SP et al. A role for NANOG in $\mathrm{G} 1$ to $S$ transition in human embryonic stem cells through direct binding of CDK6 and CDC25A. J Cell Biol 2009; 184: 67-82.

2. Neganova I, Zhang $X$, Atkinson $S$, Lako $M$. Expression and functional analysis of $G 1$ to $S$ regulatory components reveals an important role for CDK2 in cell cycle regulation in human embryonic stem cells. Oncogene 2009; 28: 20-30.

3. Neganova I, Vilella F, Atkinson SP, Lloret M, Passos JF, von Zglinicki T et al. An important role for CDK2 in G1 to $S$ checkpoint activation and DNA damage response in human embryonic stem cells. Stem Cells 2011; 29: 651-659.

4. Rohrabaugh S, Mantel C, Broxmeyer HE. Mouse hematopoietic stem cells, unlike human and mouse embryonic stem cells, exhibit checkpoint-apoptosis coupling. Stem Cells Dev 2008; 17: 1017-1020.

5. Liu W, Sun X. Skewed X chromosome inactivation in diploid and triploid female human embryonic stem cells. Human Reprod 2009; 8: 1834-1843.

6. Holubcova Z, Matula P, Sedlakova M, Vinarsky V, Dolezalova D, Barta T et al. Human embryonic stem cells suffer from centrosomal amplification. Stem Cells 2011; 29: 46-56.

7. Hyka-Nouspikel N, Desmarias J, Gokhale PJ, Jones M, Meuth M, Andrews PW et al. Deficient DNA damage response and cell cycle checkpoints lead to accumulation of point mutations in Human Embryonic. Stem Cells 2012; 30: 1901-1910.

8. Dekel-Naftali M, Aviram-Goldring A, Litmanovitch T, Shamash J, Yonath H, Hourvitz A et al. Chromosomal integrity of human preimplantation embryos at different days post fertilization. J Assist Reprod Genet 2013; 30: 633-648.

9. Zucchelli M, Ström S, Holm F, Malmgren H, Sahlén S, Religa P et al. In vivo differentiated Human Embryonic Stem Cells can acquire chromosomal aberrations more frequently than in vitro during the same period. Stem Cells Dev 2012; 21: 3363-3371.

10. Amps K, Andrews PW, Anyfantis G, Armstrong L, Avery S, Baharvand H et al. International Stem Cell Initiative. Screening ethnically diverse human embryonic stem cells identifies a chromosome 20 minimal amplicon conferring growth advantage. Nat Biotechnol 2011; 29: 1132-1144.

11. Malumbres M, Barbacid M. Cell cycle, CDKs and cancer: a changing paradigm. Nat Rev Cancer 2009; 9: 153-166.

12. Santamaría D, Barrière $C$, Cerqueira A, Hunt $S$, Tardy $C$, Newton $K$ et al. Cdk1 is sufficient to drive the mammalian cell cycle. Nature 2007; 448: 811-815.

13. Satyanarayana A, Kaldis P. Mammalian cell-cycle regulation: several Cdks, numerous cyclins and diverse compensatory mechanisms. Oncogene 2009; 28: 2925-2939.

14. Diril Mk, Ratnacaram CK, Padmakumar VC, Du T, Wasser M, Coppola V et al. Cyclin-dependent kinase 1 (Cdk1) is essential for cell division and suppression of DNA re-replication but not for liver regeneration. Proc Natl Acad Sci U S A 2012; 109: 3826-3831.

15. Berthet C, Aleem E, Coppola V, Tessarollo L, Kaldis P. Cdk2 knockout mice are viable. Curr Biol 2003; 13: 1775-1785.

16. Merrick KA, Wohlbold L, Zhang C, Allen J.J, Horiuchi D, Huskey NE et al. Switching Cdk2 on or off with small molecules to reveal requirements in Human Cell proliferation. Mol Cell 2011; 42: 624-636.

17. Li L, Wang J, Hou J, Wu Z, Zhuang Y, Lu M et al. Cdk1 interplays with Oct4 to repress differentiation of embryonic stem cells into trophectoderm. FEBS Letters 2012; 586: 4100-4107.

18. Zhang WW, Zhang XJ, Liu HX, Chen J, Ren Y H, Huang DG et al. Cdk1 is required for selfrenewal of mouse embryonic stem cells. J Cell Biochem 2011; 112: 942-948.

19. Ullah Z, Kohn MJ, Yagi R, Vassilev LT, DePamphilis ML. Differentiation of trophoblast stem cells into giant cells is triggered by 957/kip2 inhibition of CDk1 activity. Genes Dev 2008; 22: 3024-3036.

20. Van Hoof D, Munoz J, Braam SR, Pinkse M W H, Linding R, Heck AJ R et al. Phosphorylation dynamics during early differentiation of human embryonic stem cells. Cell Stem Cell 2009; 7: 214-226.

21. Becker KA, Ghule PN, Therrien JA, Lian JB, Stein JL, van Wijnen AJ et al. Self renewal of human embryonic stem cells is supported by a shortened G1 cell cycle phase. J Cell Physiol 2006; 209: 883-893.

22. Pines J, Hunter T. Human cyclins A and B are differentially located in the cell and undergo cell cycle dependent nuclear transport. J Cell Biol 1991; 115: 1-17.

23. Gavet O, Pines J. Activation of cyclin B1-Cdk1 synchronizes events in the nucleus and the cytoplasm at mitosis. J Cell Biol 2010; 189: 247-259.

24. Polo S E, Jackson SP. Dynamics of DNA damage response proteins at DNA breaks: a focus on protein modifications. Genes Dev 2011; 25: 409-433.

25. You Z, Bailis JM. DNA damage and decisions: CtIP coordinates DNA repair and cell cycle checkpoints. Trends Cell Biol 2010; 20: 402-409.

26. Chehab NH, Malikzay A, Appel M, Halazonetis TD. Chk2/hCds 1 functions as a DNA damage checkpoint in G1 by stabilizing p53. Genes Dev 2000; 14: 278-288.

27. Shieh S-Y, Ahn J, Tamai K, Taya Y, Prives C. The human homologs of checkpoint kinases Chk1 and Cds1 (Chk2) phosphorylate p53 at multiple DNA damage-inducible sites. Genes Dev 2000; 14: 289-300. 
28. Hirao A, Kong Y-Y, Matsuoka S, Wakeham A, Ruland J, Yoshida $\mathrm{H}$ et al. DNA damageinduced activation of p53 by the Checkpoint Kinase Chk2. Science 2000; 287: 1824-1827.

29. Hong $Y$, Stambrook PJ. Restoration of an absent G1 arrest and protection from apoptosis in embryonic stem cells after ionizing radiation. PNAS 2004; 101: 14443-14448.

30. Momcilović $\mathrm{O}$, Choi S, Varum S, Bakkenist C, Schatten G, Navara C. Ionizing radiation induces ataxia telangiectasia mutated-dependent checkpoint signaling and $\mathrm{G}$ (2) but not $\mathrm{G}(1)$ cell cycle arrest in pluripotent human embryonic stem cells. Stem Cells 2009; 27: 1822-1835

31. Bárta T, Vinarský V, Holubcová Z, Dolezalová D, Verner J, Pospísilová S et al. Human embryonic stem cells are capable of executing G1/S checkpoint activation. Stem Cells 2010; 28: $1143-1152$.

32. Hendzel M, Wei Y, Mancini MA, Van Hooser A, Ranalli T, Brinkley BR et al. Mitosis-specific phosphorylation of histone $\mathrm{H} 3$ initiates primarily within pericentromeric heterochromatin during G2 and spreads in an ordered fashion coincident with mitotic chromosome condensation. Chromosoma 1997; 106: 348-360.

33. Mikhailov A, Cole RW, Rieder CL. DNA damage during Mitosis in Human cells delays the Methaphase/Anaphase transition via the Spindle-Assembly Checkpoint. Current Biol 2002; 12: $1797-1806$.

34. Potapova TA, Sivakumar S, Flynn JN, Li R, Gorbsky GJ. Mitotic progression becomes irreversible in prometaphase and collapses when Wee1 and Cdc25 are inhibited. Mol Biol Cell 2011; 22: 1191-1206.

35. Hauf S, Watanable Y. Kinetochore orientation in mitosis and meiosis. Cell 2004; 119: 317-327.

36. Chouinard G, Clément I, Lafontaine J, Rodier F, Estelle Schmitt E. Cell cycle-dependent localization of CHK2 at centrosomes during mitosis. Cell Div 2013; 8: 12-22.

37. Hinchcliffe EH, Miller FJ, Cham M, Khodjakov A, Sluder G. Requirement of a centrosomal activity for cell cycle progression through $\mathrm{G} 1$ into S phase. Science 2001; 291: 1547-1550.

38. Cunha-Ferreira I, Bento I, Bettencourt-Dias M. From zero to many: Control of centriole number in development and disease. Traffic 2009; 10: 482-498.

39. Allan LA, Clarke PR. Phosphorylation of caspase- 9 by CDK1/ cyclin B1 protects mitotic cells against apoptosis. Mol Cell 2007; 26: 301-310.

40. O'Connor DS, Grossman D, Plescia J, Li F, Zhang H, Villa A et al. Regulation of apoptosis at cell division by p34cdc2 phosphorylation of survivin. Proc Natl Acad Sci U S A 2000; 97: 13103-13107.

41. Terrano DT, Upreti M, Chambers TC. Cyclin-Dependent Kinase 1-mediated Bcl-xL/Bcl-2 phosphorylation acts as a functional link coupling mitotic arrest and apoptosis. Mol Cell Biol 2010; 30: 640-656.

42. Harley ME, Allan LA, Sanderson HS, Clarke PR. Phosphorylation of Mcl-1 by CDK1-cyclin B1 initiates its Cdc20-dependent destruction during mitotic arrest. EMBO J 2010; 29 : 2407-2420.

43. Konishi Y, Lehtinen M, Donovan N, Bonni A. Cdc2 phosphorylation of BAD links the cell cycle to the cell death machinery. Mol Cell 2002; 9: 1005-1016.
44. Dumitru R, Gama V, Fagan BM, Bower JJ, Swahari V, Larysa $\mathrm{H}$ et al. Human embryonic stem cells have constitutively active Bax at the Golgi and are primed to undergo rapid apoptosis. Mol Cell 2012; 46: 573-583.

45. Shall S, de Murcia G. Poly (ADP-ribose) polymerase-1: what have we learned from the deficient mouse model? Mut Res 2000; 460: 1-15.

46. Boyer LA, Lee TI, Cole MF, Johnstone SJ, Levine SS, Zucker JP et al. Core transcriptional regulatory circuitry in human embryonic stem cells. Cell 2005; 122: 947-956.

47. Brumbaugh J, Russell JD, Yu P, Mestphall MS, Coon JJ, Thomson JA. NANOG is multiply phosphorylated and directly modified by ERK2 and CDK1 in vitro. Stem Cell Reports 2014; 2 : $18-25$.

48. Datta NS, Williams JL, Caldwell J, Curry AM, Ashcraft EK, Long MW. Novel alterations in CDK1/cyclin B1 kinase complex formation occur during the acquisition of a polyploid DNA content. Mol Biol Cell 1996; 7: 209-223.

49. L'Italien L, Tanudji M, Russell L, Schebye XM. Unmasking the redundancy between Cdk1 and Cdk2 at G2 phase in human cancer cell lines. Cell Cycle 2006; 5: 984-993.

50. Vassilev LT, Tovar C, Chen S, Knezevic D, Zhao X, Sun H et al. Selective small-molecule inhibitor reveals critical mitotic functions of human CDK1. Proc Natl Acad Sci U S A 2006; 103: $10660-10665$

51. Hochegger H, Dejsuphong D, Sonoda E, Saberi A, Eeson Rajendra E, Kirk J et al. An essential role for $\mathrm{Cdk} 1$ in $S$ phase control is revealed via chemical genetics in vertebrate cells. J Cell Biol 2007; 178: 257-268.

52. Desmarias JA, Hoffmann MJ, Bingham G, Gagou ME, Meuth M, Andrews PW et al. Human Embryonic Stem Cells Fail to Activate CHK1 and Commit to Apoptosis in Response to DNA Replication Stress. J Stem Cells 2012; 30: 1385-1393.

53. Valenzuela MT, Guerrero R, Núñez Ml, de Almodóvar JMR, Sarker M, de Murcia G et al. PARP-1 modifies the effectiveness of p53-mediated DNA damage response. Oncogene 2002; 21: 1108-1116.

(1) Cell Death and Disease is an open-access journal published by Nature Publishing Group. This work is licensed under a Creative Commons Attribution 4.0 International Licence. The images or other third party material in this article are included in the article's Creative Commons licence, unless indicated otherwise in the credit line; if the material is not included under the Creative Commons licence, users will need to obtain permission from the licence holder to reproduce the material. To view a copy of this licence, visit http://creativecommons.org/licenses/by/4.0 